\title{
Efficacy of combined treatment with oral and topical acyclovir in first episode genital herpes
}

\author{
G R KINGHORN,* I ABEYWICKREME,* M JEAVONS, ${ }^{*}$ I BARTON, $†$ CW POTTER, $†$ D JONES $\ddagger$ AND \\ E HICKMOTT $\ddagger$
}

From the *Department of Genitourinary Medicine, Royal Hallamshire Hospital, Sheffield, the $\dagger$ Department of Virology, University of Sheffield Medical School, Sheffield, and the $\ddagger$ Clinical and Applied Research Division, Wellcome Research Laboratories, Beckenham, Kent.

SUMMARY Fifty patients presenting with first episode genital herpes were randomly allocated to seven day treatment with either oral acyclovir plus $5 \%$ acyclovir cream or oral acyclovir plus matching placebo cream. Combined treatment with oral and topical acyclovir was associated with a shorter duration of itching in women alone $(p=0.04)$ but gave no clinical relief of other symptoms, the time to healing of lesions, or the subsequent recurrence rate. Concomitant topical treatment with 5\% acyclovir cream confers no advantages on patients who receive oral acyclovir.

\section{Introduction}

Acyclovir provides clinically effective antiviral treatment of first episode genital herpes when administered intravenously, ${ }^{1}$ orally, ${ }^{2-3}$ or topically. ${ }^{4}$ The results of two recent studies in Sheffield have suggested that the antiviral effect of oral acyclovir is clearly superior to that of topical acyclovir cream. ${ }^{56}$

In the study published here the objective was to assess whether the concomitant use of acyclovir cream and oral acyclovir in patients suffering from first episode genital herpes conferred any more antiviral or clinical benefit than a placebo cream and oral acyclovir.

\section{Patients and methods}

\section{STUDY POPULATION}

Men and women aged 16 years or more who presented within six days of the onset of symptoms of first episode genital herpes to the department of genitourinary medicine, Royal Hallamshire Hospital, Sheffield, were eligible to enter the study. Patients were excluded if they had used other antiviral or immune stimulation treatment within the preceding seven days, if they had underlying immune deficiency, hepatic or renal disease, or were women who were not using a valid form of contraception. All entrants to the study gave informed consent.

Address for reprints: Dr G Kinghorn, Department of Genitourinary Medicine, Royal Hallamshire Hospital, Glossop Road, Sheffield S10 2JF

Accepted for publication 12 September 1985
The efficacy of each treatment was assessed by comparing the groups for the duration of viral shedding, duration of symptoms, and the times to healing of lesions. Each variable was analysed separately by fitting Cox's proportional hazards model with adjustments made for differences in response between male and female patients and between users and non-users of co-trimoxazole.

\section{STUDY DESIGN}

All patients were treated with oral acyclovir $200 \mathrm{mg}$ four times daily for seven days. In addition they were given topical treatment with either $5 \%$ acyclovir cream or a matching placebo cream to be applied five times daily for seven days. The treatment was dispensed in a double blind fashion with separate stratification for the sex of the patient. Patients in both treatment groups were given identical advice regarding additional symptomatic treatment. Concomitant co-trimoxazole treatment was given to those patients considered to have secondarily infected lesions at presentation.

PATIENT ASSESSMENTS, LABORATORY STUDIES, AND TOXICITY SCREENING

These were identical to those described in detail in previous studies. $^{67}$

\section{FOLLOW UP FOR RECURRENCE}

After their lesions had completely healed, patients were asked to report back to the clinic in the event of any subsequent recurrence and were seen routinely three and six months after entry. 
TABLE I Comparison of treatment groups at presentation

\begin{tabular}{lcc}
\hline & Patients treated with oral acyclovir and: \\
\cline { 2 - 3 } & $\begin{array}{c}\text { Acyclovir cream } \\
(n=24)\end{array}$ & $\begin{array}{l}\text { Placebo cream } \\
(n=25)\end{array}$ \\
\hline Number of men & 7 & 7 \\
Number of women & 17 & 18 \\
Mean age (years) & $21 \cdot 5$ & $20 \cdot 7$ \\
Mean duration of symptoms (days) & $4 \cdot 4$ & $3 \cdot 0$ \\
Mean duration of lesions (days) & $3 \cdot 3$ & $16(64)$ \\
No (\%) with HSV-1 isolates & $12(50)$ & $24(96)$ \\
No $\%$ culture positive & $23(96)$ & $23(92)$ \\
No $\%$ with true primary (antibody negative) infection & $20(83)$ & $10(40)$ \\
No $(\%)$ also treated with co-trimoxazole & $12(50)$ & \\
\hline
\end{tabular}

HSV-1 = herpes simplex virus type 1 .

\section{STATISTICAL ANALYSIS}

The treatment groups were compared for their demographic and clinical data at entry to the study. Possible differences between the groups in the numbers of each sex, proportions infected with herpes simplex virus type 1 , and proportions with true primary infections, were assessed using the Fisher's Exact test. Differences in the severity of symptoms were assessed using the Pearson $\chi^{2}$ test; in the mean age of patients using the $t$ test; in the duration of symptoms and signs before presentation using the log rank test.

\section{Results}

Of 50 patients who entered the study one defaulted before completing the protocol and was excluded from the analysis. Of the 49 patients completing treaiment, 24 received acyclovir cream and 25 received placebo cream. Three, two receiving acyclovir cream and one receiving placebo cream, did not complete follow up.

Table I compares the treatment groups at entry to the study. The differences for any of the assessed demographic or clinical variables were not significant.

Table II shows the efficacy of each treatment for women only and for men and women combined. The mean (SD) duration of viral shedding, of a variety of symptoms, and of the times to healing of lesions are given. Small numbers prevented satisfactory analysis of the results for men only.

The only variable showing a statistically significant difference between the treatment groups was the shorter duration of itching in women treated with combined topical and oral acyclovir $(p=0 \cdot 04)$. All other variables gave non-significant results and no evidence of any trends.

There was no significant difference between the treatment groups in the proportion who developed one or more recurrences during the subsequent six month follow up period or in the times to first recurrence.

Of the 46 patients who completed follow up, 11 (50\%) out of 22 who received acyclovir cream compared with $10(42 \%)$ out of 24 treated with placebo

TABLE II Efficacy of treatment with oral acyclovir and either acyclovir or placebo cream

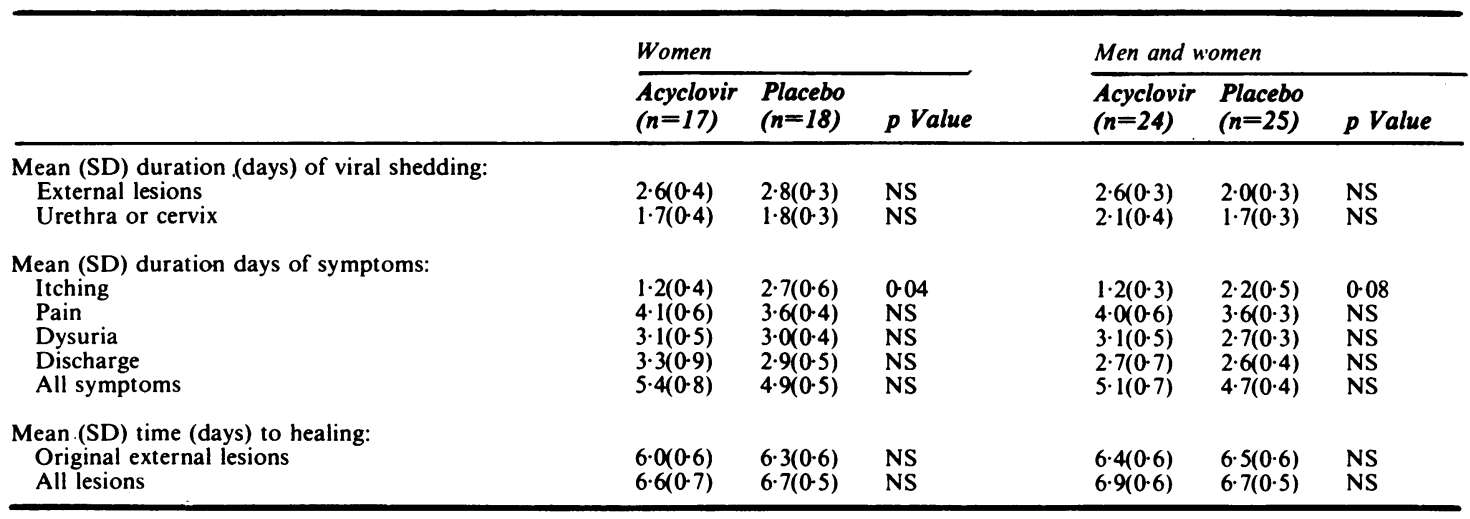


cream had a recurrence within six months of their first episode.

No adverse effects were reported by patients in any of the treatment groups, and values for haematological variables and biochemical data remained within their normal ranges after treatment.

\section{Discussion}

The durations of viral shedding and clinical manifestations observed in this study were similar to those observed in acyclovir treatment patients in a previous placebo controlled study. ${ }^{6}$ The only variable that showed a significant difference between the treatment groups was the shorter duration of itching in women given concomitant acyclovir cream than in those given placebo cream. Itching was invariably the complaint of shortest duration among our patients, and there was no evidence of benefit from concomitant topical acyclovir treatment on the other more severe and disabling symptoms that occur in first episode genital herpes. As topical treatment with acyclovir does not give any additional benefits on the duration of viral shedding, the time to healing of lesions and the subsequent recurrence rate, it is not justified in patients who receive oral acyclovir to treat first episode genital herpes.

\section{References}

1. Mindel A, Adler MW, Sutherland S, Fiddian AP. Intravenous acyclovir treatment for primary genital herpes. Lancet 1982;i:697-700.

2. Nilsen AE, Aasen R, Halsos AM, et al. Efficacy of oral acyclovir in the treatment of initial and recurrent genital herpes. Lancet 1982;ii:571-3.

3. Bryson $\mathrm{YJ}$, Dillon $\mathrm{M}$, Lovett $\mathrm{M}$, et al. Treatment of first episodes of genital herpes simplex virus infection with oral acyclovir. $N$ Engl J Med 1983;308:916-21

4. Thin RN, Nabarro JM, Davidson-Parker J, Fiddian AP. Topical acyclovir in the treatment of initial genital herpes. British Journal of Venereal Diseases 1983;59:116-9.

5. Kinghorn GR, Turner EB, Barton IG, Potter CW, Burke CA Fiddian AP. Efficacy of topical acyclovir cream in first and recurrent episodes of genital herpes. Antiviral Res 1983;3:291301 .

6. Kinghorn GR, Abeywickreme I, Jeavons M, et al. Efficacy of oral treatment with acyclovir and co-trimoxazole in first episode genital herpes. Genitourin Med 1986;62:33-7.

7. Barton IG, Kinghorn GR, Najem S, Al-Omar LS, Potter CW Incidence of herpes simplex virus types 1 and 2 isolated in patients with herpes genitalis in Sheffield. British Journal of Venereal Diseases 1982;58:44-7. 